\title{
Interactions between environmental stressors: the influence of salinity on host-parasite interactions between Daphnia magna and Pasteuria ramosa
}

\author{
Matthew D. Hall • Andrea Vettiger • \\ Dieter Ebert
}

Received: 7 June 2012/ Accepted: 24 August 2012/Published online: 22 September 2012

(C) Springer-Verlag 2012

\begin{abstract}
Interactions between environmental stressors play an important role in shaping the health of an organism. This is particularly true in terms of the prevalence and severity of infectious disease, as stressors in combination will not always act to simply decrease the immune function of a host, but may instead interact to compound or even oppose the influence of parasitism on the health of an organism. Here, we explore the impact of environmental stress on host-parasite interactions using the water flea Daphnia magna and it is obligate parasite Pasteuria ramosa. Utilising an ecologically relevant stressor, we focus on the combined effect of salinity and P. ramosa on the fecundity and survival of the host, as well as on patterns of infectivity and the proliferation of the parasite. We show that in the absence of the parasite, host fecundity and survival was highest in the low salinity treatments. Once a parasite was introduced into the environment, however, salinity and parasitism acted antagonistically to influence both host survival and fecundity, and these patterns of disease were unrelated to infection rates or parasite spore loads. By summarising the form of interactions found in the broader Daphnia literature, we highlight how the combined effect of stress and parasitism will vary with the type of stressor, the trait used to describe the health of Daphnia and the host-parasite combination under observation. Our results highlight how the context-dependent nature of interactions between stress and parasitism inevitably
\end{abstract}

Communicated by David Marcogliese.

M. D. Hall ( $₫)$ · A. Vettiger · D. Ebert

Zoologisches Institut, Evolutionsbiologie, University of Basel,

Vesalgasse 1, 4051 Basel, Switzerland

e-mail: matt.hall@unibas.ch complicates the link between environmental factors and the prevalence and severity of disease.

Keywords Bacteria - Environmental conditions -

Infection · Infectious disease · Salt stress · Stress · Virulence

\section{Introduction}

Throughout their lives animals are challenged by a range of environmental stressors that impact on the health and fitness of the individual. By definition, a stressor is any external factor that influences the ability of an organism to maintain its natural regulation or functioning to such an extent that fitness is reduced (Selye 1950; Brett 1958; but see Hoffmann and Parsons 1991); as such, a stressor can relate to an inexhaustible range of abiotic and biotic factors. Variation in environmental characteristics, such as temperature and food availability, together with the presence of predators and parasites have all been shown to impact on the physiological function and life-history of an individual (reviewed in Hofmann and Todgham 2010; Hawlena and Schmitz 2010; Flatt and Heyland 2011; Schmid-Hempel 2011). These naturally occurring stressors, however, are unlikely to act independently (i.e. additively) but instead may interact to compound or even oppose the influences of other stressors on the health of an organism (synergistic vs. antagonistic interactions, respectively). Viewing environmental stressors in isolation, therefore, can lead to a distorted picture of how individual stressors would potentially combine in natural populations to influence the health of an organism (Marcogliese and Pietrock 2011).

Of particular interest for studies of infectious disease is how the impact of parasites and pathogens is linked to 
other environmental sources of stress (Esch et al. 1975; Lafferty and Holt 2003; Marcogliese and Pietrock 2011). In the biomedical literature, it has been recognised that physical or even psychological stress can downregulate various components of the immune response (Khansari et al. 1990; Glaser et al. 1999), making individuals more susceptible to infectious disease. Environmental stressors will also influence the ability of an organism to acquire, process, and store nutrients, reducing the pool of resources that individuals can invest in disease resistance (e.g., condition dependence of immunocompetence, see Zuk and Stoehr 2002; Schmid-Hempel 2003). While the impact of environmental stress is, by definition, negative for the host, a more complex pattern may exist for the parasite. Early in the infection process, stress-induced suppression of the immune system will potentially aid the ability of a parasite to establish infection (Le Moullac et al. 1998; Malham et al. 2003). In contrast, once the parasite has evaded this initial immune response, any further stress will adversely affect host condition, decreasing the resources available for the parasite to grow and multiply within the host (Frost et al. 2008; Seppälä et al. 2008). Accordingly, both the timing and intensity of any environmental stress are important factors influencing how environmental challenges are linked to parasite fitness and the characteristics of infectious disease.

In the study reported here, we explored the impact of environmental stress on host-parasite interactions using the water flea Daphnia magna. Daphnia is increasingly being used as a model system for research into environmental stress (Eads et al. 2008) and host-parasite interactions (Ebert 2005, 2008). Studies have shown how food quality (Pulkkinen and Ebert 2004; Frost et al. 2008), maternal stress (Ben-Ami et al. 2010; Stjernman and Little 2011; Hall and Ebert 2012), temperature (Mitchell et al. 2005; Vale et al. 2008), predation (Yin et al. 2011) and pesticides (Coors et al. 2008; Coors and De Meester 2011) can all impact on host-parasite interactions. Here, we manipulated an additional stressor of Daphnia (i.e. salinity) and characterised the impact of the obligate bacterium parasite Pasteuria ramosa on host traits. For D. magna of coastal rock-pool habitats, salinity is an important environmental stressor. At our study location, peak fluctuations in salinity have been recorded up to 12.5 parts per thousand (approximately $12.5 \mathrm{~g} \mathrm{~L}^{-1}$ ), although normal variation between rock pools is much lower [mean \pm standard deviation (SD) parts per thousand: $1.97 \pm 1.47$, as summarised in Ranta 1979]. Such variation affects the overall physiological condition of individuals by influencing a range of key processes, such as feeding, swimming speed, metabolism, growth, reproduction and survival (Arnér and Koivisto 1993; Baillieul et al. 1998). Despite the importance of salinity as an environmental stressor for rock-pool populations of Daphnia, however, the link between salinity and parasitism has not been directly explored, although studies have explored such interactions in other crustaceans (Dunn and Hatcher 1997; Piscart et al. 2007; Studer and Poulin 2012).

By exposing a single clone of D. magna to four salt concentrations (0.33-3 $\mathrm{g} \mathrm{L}^{-1}$ as commercial sea salt) and manipulating the presence or absence of the parasite $P$. ramosa, we aimed to understand how a natural stressor, salinity, can impact host-parasite interactions. In our study, we focused exclusively on the within-host dynamics of infection. To achieve this we first used a compatible combination of host and parasite clones (Luijckx et al. 2010). Second, we manipulated the salt concentration only after the individuals were exposed to the parasite. In this way we excluded the potential effects of stress on feeding or other behaviours, which may reduce the encounter rates between the parasite and the host, and explored the impact of stress directly on host physiology. We then measured characteristics of both host and parasite fitness, such as survival, offspring production, infectivity and spore production. In doing so, the aim of our study was to address three important questions: (1) how does host performance change along a salinity gradient; (2) how does the interaction between salinity and $P$. ramosa infection compare to the broader patterns of environmental stress and parasitism in the Daphnia literature; (3) can any differences in the severity of disease be accounted for by changes in the growth or infectivity of the parasite along a salinity gradient?

\section{Materials and methods}

Host and parasite clones

The host Daphnia magna Straus is a freshwater cladoceran that reproduces through cyclical parthenogenesis and inhabits eutrophic shallow ponds throughout Eurasia. The parasite Pasteuria ramosa Metchnikoff 1888 is a bacterial obligate endoparasite of Daphnia that causes severe loss of host fitness via castration and reduced survival (Ebert et al. 2004). Following infection, the parasite grows within the body cavity of its host, producing endospores that give the infected individual a distinctive reddish-brown colouration. Transmission only occurs horizontally with the spores released from the decaying cadaver of a formerly infected host.

For our study, all host individuals were derived from a single D. magna clone (SP1-2-3) originating from a rockpool on the island of Spikarna, Tvärminne archipelago, in south-western Finland. In this small coastal rock-pool habitat, salinity can change within very short periods as the 
result of a sudden invasion of Baltic Sea water or as a consequence of strong rain. The parasite clone (C19) originated from single spore infection based on an isolate (P1) of $P$. ramosa collected from Germany (Luijckx et al. 2010). This parasite is naturally infective to D. magna clone SP1-2-3 and resembles $P$. ramosa clones from the same rock-pool habitat (Luijckx et al. 2010). Parasite suspensions were produced by grinding up infected D. magna and adjusting the spore density to the desired concentration. A placebo suspension was produced with the same amount of uninfected Daphnia.

\section{Parasite and salinity challenges}

We used a split-brood design where individuals from a single mother were split across the two parasite treatments (exposed and unexposed) and the four salinity treatments (0.33-3 $\left.\mathrm{g} \mathrm{L}^{-1}\right)$, resulting in eight treatment combinations. Each treatment comprised 35 individual replicates (280 individuals in total), although nine individuals, distributed evenly across the treatments, were subsequently excluded as they died before the salinity manipulation could be applied. The saline concentrations were obtained by mixing commercial sea salt (hW-Meersalz; Wiegandt $\mathrm{GmbH}$, Krefeld, Germany) in $5 \mathrm{~L}$ of artificial medium (ADaM; Klüttgen et al. 1994; modified after Ebert et al. 1998). Each time we created four salinity concentrations from $0.33 \mathrm{~g}$ $\mathrm{L}^{-1}$ (the normal salinity of ADaM) and then 1,2 or $3 \mathrm{~g}$ $\mathrm{L}^{-1}$. Beginning with 3-day-old Daphnia placed individually in $100-\mathrm{mL}$ jars filled with $20 \mathrm{~mL}$ of normal ADaM $\left(0.33 \mathrm{~g} \mathrm{~L}^{-1}\right)$, we first exposed individuals to the parasite and then applied the salinity challenge. At ages 5 and 6 days, animals received 5,000 spores of the C19 $P$. ramosa clone $(10,000$ in total) or the equivalent volume of the placebo suspension as the control. At age 7 days, we moved individuals to new jars and replaced the standard (low salinity) medium with $100 \mathrm{~mL}$ of the appropriate experimental saline concentrations.

Thereafter, every 3 days (i.e. ages 10, 13, 16 days, etc.) we moved individuals to new jars containing fresh medium of the appropriate salinity treatment and counted the number of offspring produced. Individuals were monitored daily for survival until 30 days post-exposure to the parasite, at which point all Daphnia were individually frozen $\left(-20^{\circ} \mathrm{C}\right)$ for the later assessment of infection status and spore production. We counted the number of mature transmission stage spores by crushing the Daphnia cadavers and counting two independent samples of this suspension using a Neubauer improved counting chamber (depth $0.1 \mathrm{~mm}$ ). Throughout the experiment we maintained individuals in a single climate-controlled incubator (light/ dark: $16 / 8 \mathrm{~h} ; 20 \pm 0.5^{\circ} \mathrm{C}$ ) and regularly shuffled the location of the individuals to equalise any positional effects. To account for the growing demands of the animals, food levels were increased gradually, beginning on day 2 with $0.5 \times 10^{6}$ algae cells of Scenedesmus obliquus per Daphnia per day, and increasing to $1 \times 10^{6}, 2 \times 10^{6}$, $2.5 \times 10^{6}, 3 \times 10^{6}$ and $8 \times 10^{6}$ algal cells per animal per day on days $4,7,9,11$ and 13 , respectively.

\section{Statistical analysis}

We recorded survival and fecundity for 136 control individuals (33-35 individuals per treatment) and survival, fecundity, infection status and spore loads for 135 individuals exposed to $P$. ramosa (32-35 per treatment). In the subsequent analysis, all individuals were used for the estimates of host survival (control and exposed individuals) and infectivity (exposed individuals only). However, to avoid confounding other estimates of host and parasite performance with survival, only individuals that survived until the end of the experiment (day 30 post-exposure to $P$. ramosa) were used for estimates of host offspring production and parasite spore count. Specifically, we included 104 control individuals (23-31 individuals per treatment) and 75 infected individuals (15-21 individuals per treatment). We thus refer to the parasitism treatment as "parasite exposure" for host survival (as individuals may or may not have been infected when they died) and "parasite infection" for host offspring production (as individuals could be reliably identified as infected at day 30 postexposure).

All statistical analyses were performed in R (ver. 2.12.2; R Development Core Team, available at: www.R-project. org). For host survival and host offspring production, we assessed the effect of both parasite exposure/infection and salinity using a full-factorial model where salinity, parasite exposure/infection and their interaction were included as fixed effects. For the parasite traits, infection status and spore counts, only salinity was included as a fixed effect. We analysed binomial data, such as host survival and infection status, using a generalised linear model with a binomial error link function. Before analyses, we tested for the assumptions of the linear (normality, homogeneity of variances) and generalised linear (over-dispersion) models. In the case of the offspring production data, there was significant heterogeneity in variances between the treatments groups (Levene's test, $P<0.001$ ). Accordingly, we used a white-adjusted analysis of variance to correct for unequal variances as implement using the car package of $\mathrm{R}$ (Fox and Weisberg 2011). We also transformed spore load using a natural log transformation, although the figures here are presented on the original scale. Finally, treatment effects were explored post hoc using pairwise comparisons, based on Student's $t$ tests and chi-square tests for the linear and generalised linear models, respectively. 


\section{Results}

We first examined the impact of salinity alone and salinity in combination with parasite exposure on the survival of D. magna over the period of experiment (Fig. 1). All individuals from the treatment groups were included in this analysis, irrespective of the infection status of exposed individuals. There was a significant effect of parasite exposure $\left(\chi_{1}^{2}=9.072, P=0.003\right)$ and an interaction between salt concentration and parasite exposure $\left(\chi_{3}^{2}=10.111, P=0.018\right)$ on host survival. There was no independent effect of salt concentration alone $\left(\chi_{3}^{2}=1.819\right.$, $P=0.611)$. Figure 1 reveals how the interaction between salinity and parasite exposure influences the patterns of host survival. Only at the lowest salt concentration $(0.33 \mathrm{~g}$ $\mathrm{L}^{-1}$ ) was there a significant difference between exposed and non-exposed individuals, with the survival for both treatment groups converging as the salt concentrations increase.

We then examined the combined effect of salinity and parasite infection on the number of offspring produced by individuals that survived until the end of the experiment (30 days post-exposure). We found that offspring production was influenced by both parasite infection $\left(F_{1,170}=\right.$ 1,943.188, $P<0.001)$ and an interaction between salt concentration and parasite infection $\left(F_{3,170}=9.567\right.$,

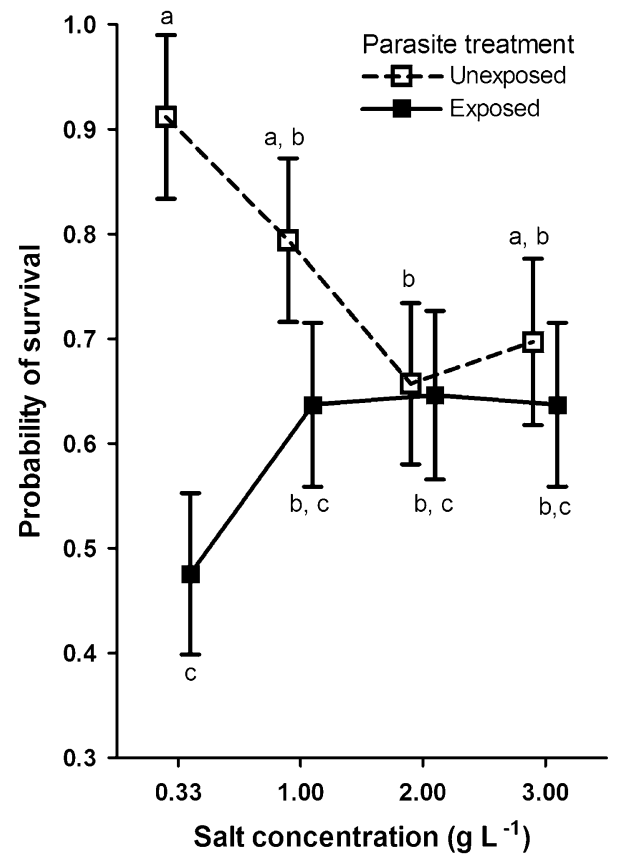

Fig. 1 The impact of salinity and parasite exposure on host survival [mean \pm standard error (SE)] over the duration of the experiment. Estimates of host survival for the parasite exposure treatment included all individuals irrespective of their infection status. Lowercase letters indicate significant groupings by post hoc comparisons using the chi-square test $(P<0.05)$
$P<0.001)$. There was no independent effect of salt concentration alone on offspring production above all other factors $\left(F_{3,170}=1.060, P=0.368\right)$. As shown in Fig. 2, individuals that were exposed to the parasite and become infected produced significantly fewer offspring than those unexposed individuals who received only the salinity manipulation, with the difference between the two groups being greatest in the low salinity treatments (0.33-1.00 $\left.\mathrm{g} \mathrm{L}^{-1}\right)$.

Finally, we assessed the impact of salinity on parasite fitness based on estimates of both infectivity and the proliferation of the parasite within the host. Infection rates were unaffected by the salinity treatment $\left(\chi_{3}^{2}=0.772\right.$, $P=0.856)$ and appeared to remain relatively constant across the salt gradients (Fig. 3a). The spore load of infected individuals, however, was influenced by the salt concentration $\left(F_{3,71}=4.063, P=0.010\right)$. The number of mature spores present in individuals that survived until the end of the experiment (30 days post-exposure) was lowest for intermediate salt concentrations (Fig. 3b).

\section{Discussion}

Interactions between parasites and other environmental stressors have increasingly been identified as playing an

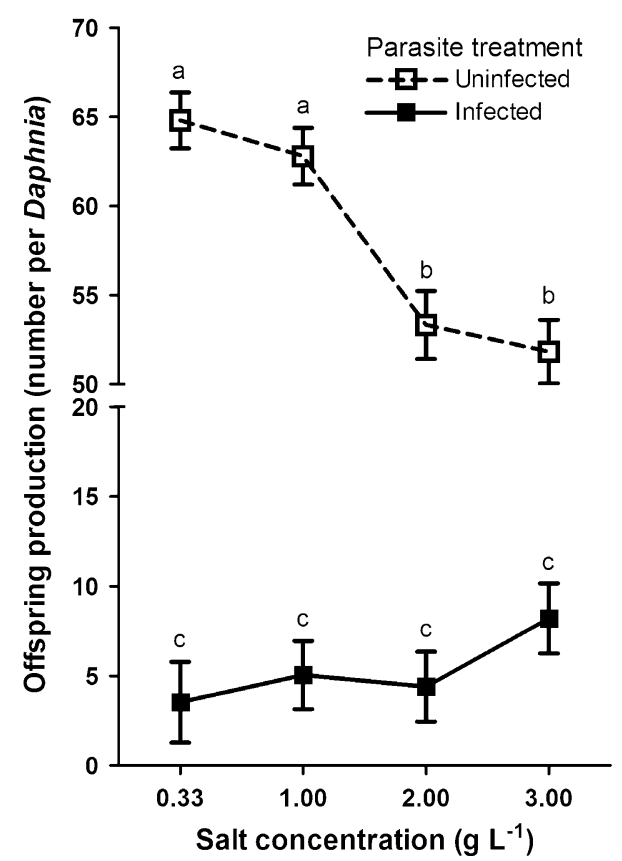

Fig. 2 The impact of salinity and parasite infection on host offspring production (mean $\pm \mathrm{SE}$ ). Confounding of fecundity and survival estimates was avoided by only including those individuals that survived until the end of the experiment in the final estimates of offspring production. Lowercase letters indicate significant groupings by post hoc comparisons using Student's $t$ test $(P<0.05)$ 

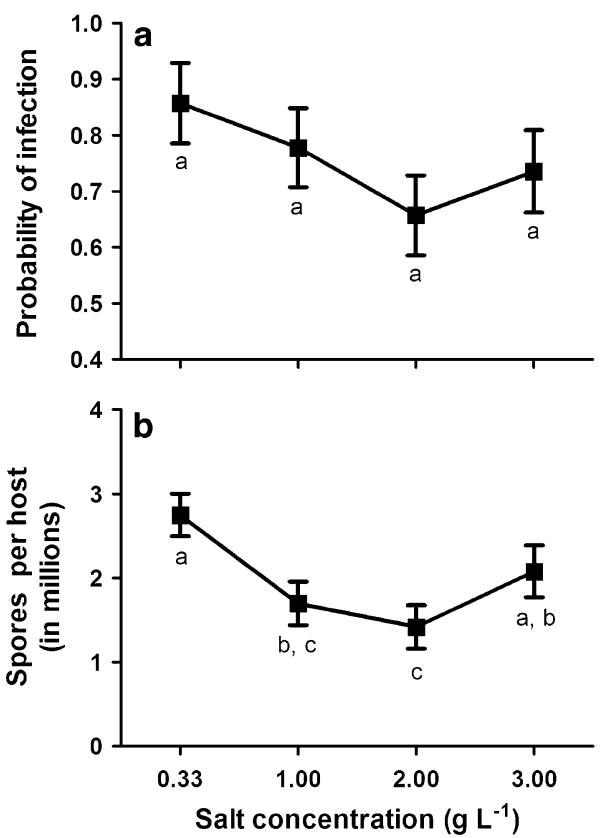

Fig. 3 The impact of salinity on infection rates (a) and spore loads (b) of $P$. ramosa. Only infected individuals that survived until the end of the experiment were included in the final estimates of spore production. For each panel, lowercase letters indicate significant groupings by post hoc comparisons using the chi-square test $(P<0.05)$ and Student's $t$ test $(P<0.05)$ for infection rates and spore loads, respectively. Data are presented as the mean $\pm \mathrm{SE}$

important role in shaping the health of an organism (reviewed in Marcogliese and Pietrock 2011). In our study, we focussed on the link between stress and infectious disease by manipulating the presence of the parasite $P$. ramosa and then exposing individuals of D. magna to a salinity gradient. Consistent with a range of other studies (Gonçalves et al. 2007; Martínez-Jerónimo and MartínezJerónimo 2007; Heine-Fuster et al. 2010), we found a decrease in host fitness at higher salt concentrations. In the absence of the parasite, host fitness was highest at the lower end of the salinity gradient, both in terms of survival (Fig. 1) and reproductive output (Fig. 2). Once a parasite was introduced into the environment, however, our results show that the correlation between salinity and host fitness changed considerably.

For the Daphnia and P. ramosa host-parasite system, the impact of parasitism is expected to reduce host survival and fecundity (Ebert et al. 2004). In our study, exposure to $P$. ramosa did not simply increase the average impact of salinity on host reproduction and survival (i.e. acted additively), rather it interacted antagonistically with salinity to modify the patterns of host fitness. In terms of host survival, the significant interaction between salinity and parasite exposure reflects that survival between unexposed and exposed individuals differed only at the lower end of the salinity gradient (Fig. 1). The interaction term for host reproduction, in contrast, arises because the reproductive output of infected individuals remained constant across the salinity gradient (Fig. 2). Such antagonistic interactions have also been found in the amphipod Gammarus roeseli, where infection by an acanthocephalan parasite acted to increase the salinity tolerance of the host (Piscart et al. 2007). Antagonism between salinity and parasitism, however, is not necessarily universal, as other studies have found no clear evidence for an interaction between salinity and parasitism on traits such as clutch size (Dunn and Hatcher 1997) and survival (Sousa and Gleason 1989; Reisser and Forward 1991).

Indeed, context-dependent interactions between stressors appear to be a common feature of host-parasite interactions. To highlight this point, a summary of multiple studies across different Daphnia-parasite combinations is presented in Table 1 (this study; Bittner et al. 2002; Lass and Bittner 2002; Ebert et al. 2004; Coors and De Meester 2008; Frost et al. 2008; Vale et al. 2008; Coors et al. 2008; Yin et al. 2011). For each host trait and host-parasite combination, we describe the general impact of the environmental manipulation on host traits in the presence and absence of the parasite and classify the impact of multiple stressors as either additive, synergistic or antagonistic. From this summary, it is clear that all possible forms of interactions can be found in studies investigating stress and parasitism in Daphnia and that certain host-parasite combinations may also respond differently to a given stressor. In Table 1, for example, the interaction between pesticide exposure and parasitism was found to be additive for the timing and size of the first clutch but synergistic for host survival. Conversely, in Table 1 the effect of predator kairomones and the parasite Metschnikowia on the clutch size of $D$. longispina was additive, whereas for $P$. ramosa and the clutch size of D. magna it was antagonistic. Thus, the combined effect of stress and parasitism is neither specific to the host-parasite combination, the host trait of interest, nor to the type of environmental stress.

Underlying these different interactions between stressors may be some fundamental aspects of the biology of hostparasite interactions. Synergistic interactions could arise if the environment interfered directly with the ability of the host to cope with infection, whereas antagonistic interactions would occur if the environment interferes with the ability of the parasite to exploit the host. Interactions between stressors may also reflect the priorities placed on a given trait by both hosts and parasites. Under stressful conditions a host may maintain its investment in fecundity at the expense of survival, resulting in a synergistic impact of the parasite on survival under poor conditions. In our study, the antagonistic interactions between salinity and parasitism suggest that the physiological condition of a host is related to the overall severity of disease (i.e. 


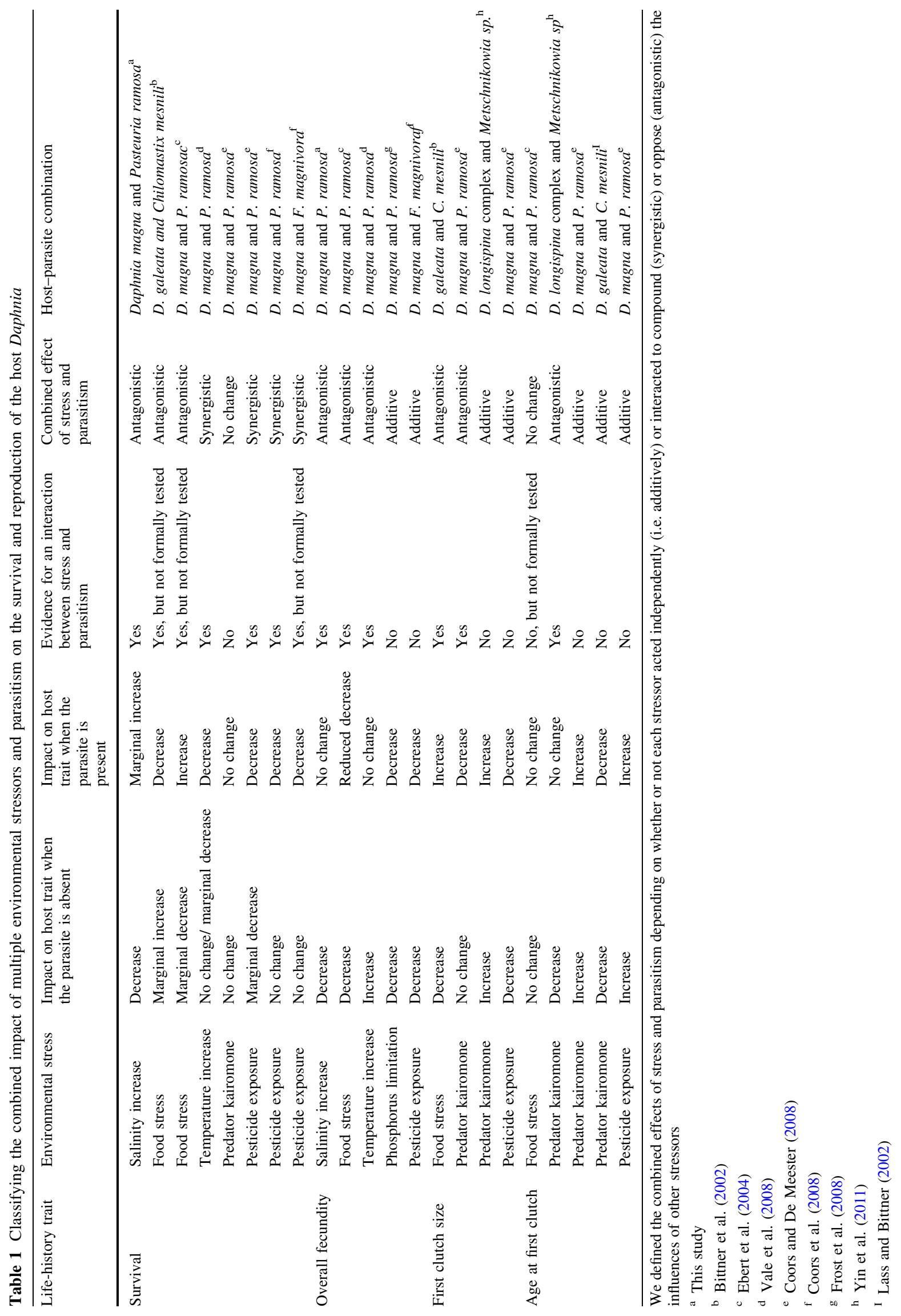


condition-dependence of virulence; Krist et al. 2004; Restif and Kaltz 2006). For both survival and fecundity, differences between the control/uninfected and exposed/infected treatments were greatest in the low salinity treatment (i.e. $0.33 \mathrm{~g} \mathrm{~L}^{-1}$ ). Therefore, the virulence of $P$. ramosa appears to depend on host condition, with the greatest reduction in host fitness occurring in the region of the salinity gradient where unexposed individuals performed best (Figs. 1, 2).

The effect of salinity on both host condition and the severity of disease, however, was not directly correlated with the prevalence of disease or the growth of the parasite (Fig. 3). Infection rates remained constant across the salinity gradient, a result that was unexpected given that host susceptibility to $P$. ramosa has been shown to depend on a range of environmental stressors (Frost et al. 2008; Vale et al. 2008; Allen and Little 2010; Hall and Ebert 2012). While studies in other host-parasite systems have also documented the lack of a correlation between salinity and host susceptibility (e.g. the amphipod Pagurus novizealandiae; Studer and Poulin 2012), contributing to our findings may have been the timing of the infection process at which the salinity treatment was applied. By using a compatible host-parasite combination and exposing Daphnia to the parasite before manipulating the salinity, we may have missed the window in which stress can impact on the ability of a host to clear a parasite infection. As such, we predict that the timing of the environmental stress to impact on the ability of a $P$. ramosa to colonise Daphnia (as opposed to influencing parasite growth) may only be a matter of hours.

Nonetheless, the timing of the salinity application would not explain the non-linear effect of salinity on parasite spore loads. The proliferation of the parasite within the host was associated with a small, but significant decrease in parasite spore loads associated with intermediate salinity concentrations. In the Daphnia-Pasteuria system, other non-linear relationships have previously been reported for temperature and parasite growth (Vale et al. 2008; Vale and Little 2009). In this case, the authors suggest that higher parasite growth at intermediate temperatures is driven by the relationship between Daphnia feeding rates and temperature (and presumably nutrient acquisition). Without a physiological understanding of how salinity impacts on both Daphnia and Pasteuria, however, it is difficult to explain why P. ramosa performs better at the extremes of a salinity gradient. Instead, we can point to the growing evidence that interactions between salinity and parasitism may be inherently complex. Short- versus long-term changes in salinity (hours compared to weeks), for example, can differentially influence the production of parasite transmission stages (Koprivnikar and Poulin 2009; Lei and Poulin 2011), and parasites may even be able to manipulate the osmoregulation ability of their host (Brooks and Mills 2011).
In summary, based on our results and a growing number of studies in both Daphnia (see Table 1) and other organisms (reviewed in Marcogliese and Pietrock 2011), it is clear that the presence of a parasite can modify the impact of additional stressors that may be present in the environment. Such trait-specific interactions are not only limited to the traits of the host, but they are also reflected in other characteristics of disease, such as the ability of a parasite to colonise and grow within the host. In particular, the timing of the stress and whether the immune system/ physiological condition of the host is affected will differentially shape the characteristics of disease. The contextdependent nature of stress and host-parasite interactions, therefore, inevitably complicates how the environment is understood to influence the evolution of virulence, as well as the predictability for which environmental factors can be linked to the prevalence and severity of disease.

Acknowledgments This study was supported by an EU Marie Curie Incoming International Fellowship (PIIF-GA-2009-252417) to MDH and by the Swiss National Science Foundation. We thank David J. Marcogliese, two anonymous reviewers and members of the Ebert Group for comments on the manuscipt, and J. Hottinger and U. Stiefel for laboratory assistance. All experiments complied with the current laws of Switzerland.

\section{References}

Allen DE, Little TJ (2010) Identifying energy constraints to parasite resistance. J Evol Biol 21:224-229

Arnér M, Koivisto S (1993) Effects of salinity on metabolism and life history characteristics of Daphnia magna. Hydrobiologia 259:69-77

Baillieul M, De Wachter B, Blust R (1998) Effect of salinity on the swimming velocity of the water flea Daphnia magna. Physiol Zool 71:703-707

Ben-Ami F, Ebert D, Regoes RR (2010) Pathogen dose infectivity curves as a method to analyze the distribution of host susceptibility: a quantitative assessment of maternal effects after food stress and pathogen exposure. Am Nat 175:106-115

Bittner K, Rothhaupt K-O, Ebert D (2002) Ecological interactions of the microparasite Caullerya mesnili and its host Daphnia galeata. Limnol Oceanogr 47:300-305

Brett JR (1958) Implications and assessment of environmental stress. In: Larkin PA (ed) The investigation of fish-power problems. University of British Columbia, Vancouver, pp 69-83

Brooks SJ, Mills CL (2011) Parasitic infection manipulates sodium regulation in the freshwater amphipod Gammarus pulex (L.). Comp Biochem Physiol A Mol Integr Physiol 159:247-252

Coors A, De Meester L (2008) Synergistic, antagonistic and additive effects of multiple stressors: predation threat, parasitism and pesticide exposure in Daphnia magna. J Appl Ecol 45:1820-1828

Coors A, De Meester L (2011) Fitness and virulence of a bacterial endoparasite in an environmentally stressed crustacean host. Parasitology 138:122-131

Coors A, Decaestecker E, Jansen M, De Meester L (2008) Pesticide exposure strongly enhances parasite virulence in an invertebrate host model. Oikos 117:1840-1846

Dunn AM, Hatcher MJ (1997) The effect of salinity on transovarial transmission of a microsporidian infecting Gammarus duebeni. Parasitology 115:381-385 
Eads BD, Andrews J, Colbourne JK (2008) Ecological genomics in Daphnia: stress responses and environmental sex determination. Heredity 100:184-190

Ebert D (2005) Ecology, epidemiology, and evolution of parasitism in Daphnia. Available at: http://www.ncbi.nlm.nih.gov/entrez/query. fcgi?db=Books. National Library of Medicine (U.S.), National Center for Biotechnology Information, Bethesda

Ebert D (2008) Host-parasite coevolution: insights from the Daphniaparasite model system. Curr Opin Microbiol 11:290-301

Ebert D, Zschokke-Rohringer CD, Carius HJ (1998) Within-and between-population variation for resistance of Daphnia magna to the bacterial endoparasite Pasteuria ramosa. Proc R Soc Lond B 265:2127-2134

Ebert D, Carius HJ, Little T, Decaestecker E (2004) The evolution of virulence when parasites cause host castration and gigantism. Am Nat 164[Suppl]:S19-S32

Esch GW, Gibbons JW, Bourque JE (1975) An analysis of the relationship between stress and parasitism. Am Midl Nat 93:339-353

Flatt T, Heyland A (2011) Mechanisms of life history evolution: the genetics and physiology of life history traits and trade-offs. Oxford University Press, Oxford

Fox J, Weisberg S (2011) An R companion to applied regression. Sage, Thousand Oaks

Frost PC, Ebert D, Smith VH (2008) Responses of a bacterial pathogen to phosphorus limitation of its aquatic invertebrate host. Ecology 89:313-318

Glaser R, Rabin B, Chesney M, Cohen S, Natelson B (1999) Stressinduced immunomodulation: implications for infectious diseases? JAMA 281:2268-2270

Gonçalves AMM, Castro BB, Pardal MA, Gonçalves F (2007) Salinity effects on survival and life history of two freshwater cladocerans (Daphnia magna and Daphnia longispina). Ann Limnol-Int J Lim 43:13-20

Hall MD, Ebert D (2012) Disentangling the influence of parasite genotype, host genotype and maternal environment on different stages of bacterial infection in Daphnia magna. Proc R Soc Lond B 279:3176-3183

Hawlena D, Schmitz OJ (2010) Physiological stress as a fundamental mechanism linking predation to ecosystem functioning. Am Nat 176:537-556

Heine-Fuster I, Vega-Retter C, Sabat P, Ramos-Jiliberto R (2010) Osmoregulatory and demographic responses to salinity of the exotic cladoceran Daphnia exilis. J Plankton Res 32:1405-1411

Hoffmann AA, Parsons PA (1991) Evolutionary genetics and environmental stress. Oxford University Press, Oxford

Hofmann GE, Todgham AE (2010) Living in the now: physiological mechanisms to tolerate a rapidly changing environment. Annu Rev Physiol 72:127-145

Khansari DN, Murgo AJ, Faith RE (1990) Effects of stress on the immune system. Immunol Today 11:170-175

Klüttgen B, Dülmer U, Engels M, Ratte HT (1994) ADaM, an artificial freshwater for the culture of zooplankton. Water Res 28:743-746

Koprivnikar J, Poulin R (2009) Effects of temperature, salinity, and water level on the emergence of marine cercariae. Parasitol Res 105:957-965

Krist AC, Jokela J, Wiehn J, Lively CM (2004) Effects of host condition on susceptibility to infection, parasite developmental rate, and parasite transmission in a snail-trematode interaction. J Evol Biol 17:33-40

Lafferty KD, Holt RD (2003) How should environmental stress affect the population dynamics of disease? Ecol Lett 6:654-664

Lass S, Bittner K (2002) Facing multiple enemies: parasitised hosts respond to predator kairomones. Oecologia 132:344-349

Le Moullac G, Soyez C, Saulnier D, Ansquer D, Avarre JC, Levy P (1998) Effect of hypoxic stress on the immune response and the resistance to vibriosis of the shrimp Penaeus stylirostris. Fish Shellfish Immunol 8:621-629

Lei F, Poulin R (2011) Effects of salinity on multiplication and transmission of an intertidal trematode parasite. Mar Biol 158:995-1003

Luijckx P, Ben-Ami F, Mouton L, Du Pasquier L, Ebert D (2010) Cloning of the unculturable parasite Pasteuria ramosa and its Daphnia host reveals extreme genotype-genotype interactions. Ecol Lett 14:125-131

Malham SK, Lacoste A, Gélébart F, Cueff A, Poulet SA (2003) Evidence for a direct link between stress and immunity in the mollusc Haliotis tuberculata. J Exp Zool 295A:136-144

Marcogliese DJ, Pietrock M (2011) Combined effects of parasites and contaminants on animal health: parasites do matter. Trends Parasitol 27:123-130

Martínez-Jerónimo F, Martínez-Jerónimo L (2007) Chronic effect of $\mathrm{NaCl}$ salinity on a freshwater strain of Daphnia magna Straus (Crustacea: cladocera): a demographic study. Ecotoxicol Environ Saf 67:411-416

Mitchell SE, Rogers ES, Little TJ, Read AF (2005) Host-parasite and genotype-by-environment interactions: temperature modifies potential for selection by a sterilizing pathogen. Evolution 59: $70-80$

Piscart C, Webb D, Beisel JN (2007) An acanthocephalan parasite increases the salinity tolerance of the freshwater amphipod Gammarus roeseli (Crustacea: gammaridae). Naturwissenschaften 94:741-747

Pulkkinen K, Ebert D (2004) Host starvation decreases parasite load and mean host size in experimental populations. Ecology $85: 823-833$

Ranta E (1979) Niche of Daphnia species in rock pools. Arch Hydrobiol 87:205-223

Reisser CE, Forward RB (1991) Effect of salinity on osmoregulation and survival of a rhizocephalan parasite, Loxothylacus panopaei, and its crab host, Rhithropanopeus harrisii. Estuaries 14:102-106

Restif O, Kaltz O (2006) Condition-dependent virulence in a horizontally and vertically transmitted bacterial parasite. Oikos 114:148-158

Schmid-Hempel P (2003) Variation in immune defence as a question of evolutionary ecology. Proc R Soc Lond B 270:357-366

Schmid-Hempel P (2011) Evolutionary parasitology: the integrated study of infections, immunology, ecology, and genetics. Oxford University Press, Oxford

Selye H (1950) Stress and the general adaptation syndrome. Br Med J $1: 1383-1392$

Seppälä O, Liljeroos K, Karvonen A, Jokela J (2008) Host condition as a constraint for parasite reproduction. Oikos 117:749-753

Sousa WP, Gleason M (1989) Does parasitic infection compromise host survival under extreme environmental conditions? The case for Cerithidea californica (Gastropoda: prosobranchia). Oecologia 80:456-464

Stjernman M, Little TJ (2011) Genetic variation for maternal effects on parasite susceptibility. J Evol Biol 24:2357-2363

Studer A, Poulin R (2012) Effects of salinity on an intertidal hostparasite system: is the parasite more sensitive than its host? J Exp Mar Biol Ecol 412:110-116

Vale PF, Little TJ (2009) Measuring parasite fitness under genetic and thermal variation. Heredity 103:102-109

Vale PF, Stjernman M, Little TJ (2008) Temperature-dependent costs of parasitism and maintenance of polymorphism under genotypeby-environment interactions. J Evol Biol 21:1418-1427

Yin M, Laforsch C, Lohr JN, Wolinska J (2011) Predator-induced defense makes Daphnia more vulnerable to parasites. Evolution 65:1482-1488

Zuk M, Stoehr AM (2002) Immune defense and host life history. Am Nat 160:S9-S22 